\title{
Weakly Sentinel involving a Navier-Stokes Problem and Detecting Pollution
}

\author{
REZZOUG Imad *1 and AYADI Abdelhamid ${ }^{2}$ \\ ${ }^{1,2}$ Larbi Ben M'hidi University, Faculty of Science. Laboratory of Dynamical Systems and Control; \\ 1,2 P.O.Box 358, OEB, Algeria \\ 1 rezzoug.imad@univ-oeb.dz, ${ }^{2}$ facmaths@yahoo.fr
}

\begin{abstract}
In this paper, we consider a Navier-Stokes system with missing initial data condition and perturbuted term or pollution term. The notion of sentinel is introduced in the study of the problems with incomplete data by J.L.Lions [12]. The construction of sentinel is based on the existence and uniqueness of the adjoint system solution which is exactly controllability. In this work, we introduced the weakly sentinel can be associated to this system and allows to characterize this pollution.
\end{abstract}

Keywords: Controllability, Sentinels, Navier-Stokes system.

2010 MSC No: 93B05, 93C20, 92D40.

\section{Introduction}

\section{Abridged English version}

\subsection{Statement of the problem}

The notion of sentinel was introduced by J. L. Lions to study systems of incomplete data [12]. The notion permits to distinguish and to analysis two types of incomplete data: the so called pollution terms on which we look for information's, independently of the other type of incomplete data which is the missing terms, and that we do not want to identify.

Typically, the Lions' sentinel is a functional defined from an open set $\mathcal{O}$ on which we consider three functions: the "observation" $y_{o b s}$ corresponding to measurements, a given "mean" function $h_{0}$, and a control function $u$ to be determined.

Let us remind that Lions' sentinel theory [12] relies on the following three features: the state equation $y$ which is governed by a system of PDE, the observation system and some particular evaluation function: the sentinel itself.

For $n=\{2 ; 3\}$, let $\Omega$ be a bounded open subset of $\mathbb{R}^{n}$ with boundary $\partial \Omega=\Gamma$ of class $\mathcal{C}^{2}, T>0$. Set $\mathcal{Q}=\Omega \times(0, T)$, $\Sigma=\Gamma \times(0, T), \mathcal{U}=\mathcal{O} \times(0, T)$. We consider the perturbed system

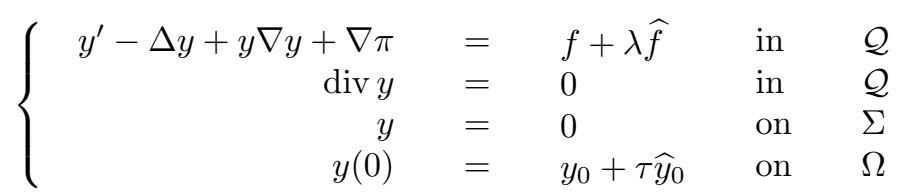

\footnotetext{
${ }^{*}$ Corresponding author. REZZOUG Imad

1 rezzoug.imad@univ-oeb.dz
} 
is a modeling transportation of a flow $y(x, t)$ located in the spatial point $x$ of the domain $\Omega$ and at the time $t \in[0, T]$. Moreover, $y$ is submitted to the pressure $\pi(x, t)$ and also to different exterior strengths represented by a source term $f$.

Where $(.)^{\prime}$ is the partial derivative with respect to time $t$. The functions $y_{0}$ and $f$ are known. But, the terms: $\tau \widehat{y}_{0}$ (so-called missing term) and $\lambda \widehat{f}$ (so-called pollution term) are unknown, $\widehat{y}_{0}$ and $\widehat{f}$ are renormalized and represent the size of missing and pollution

$y_{0}, \widehat{y}_{0} \in H ; f, \widehat{f} \in L^{2}(0, T ; H)$ or $f, \widehat{f} \in L^{2}\left(0, T ; V^{\prime}\right), V^{\prime}=$ dual of $V$ and that the reals $\lambda$ and $\tau$ are small enough.

Such that

$$
\begin{gathered}
V=\left\{\varphi / \varphi \in\left(H_{0}^{1}(\Omega)\right)^{n}, \operatorname{div} \varphi=0\right\} \\
H=\left\{\varphi / \varphi \in\left(L^{2}(\Omega)\right)^{n}, \operatorname{div} \varphi=0 ; \varphi \cdot \nu=0 \quad \text { on } \quad \Gamma\right\}
\end{gathered}
$$

The observation is $y$ on $\mathcal{O}$, for the time $T$. we denote by $y_{o b s}$ this observation

$$
y_{\text {obs }}=m_{0} \in\left(L^{2}(\mathcal{O} \times] 0, T[)\right)^{n}
$$

the problem (1) admits a unique solution in $L^{2}(0, T ; V) \cap L^{\infty}(0, T ; H)$. For the sake of simplicity, we denote $y(x, t ; \lambda, \tau)=y(\lambda, \tau)$.

The general question we want to address is

(q): given some observation of the state of system, can one obtain $\lambda \widehat{f}$ without any attempt at computing $\tau \widehat{y}_{0}$ ?

Least squares. Question (q) is natural and lids to some developments; some answer is given by the least squares method. The method consists in considering the unknowns \{pollution term; missing term $\}=\{v, w\}$ as control variables, then the state $y(x, t ; v, w)$ has to be driven as close as possible to $m_{0}$.

This comes to some optimal control problem. By this way we look for the pair $(v ; w)$, there is then no real possibility to find $v$ or $w$ independently.

Sentinels. The sentinel method of Lions [12] is a particular least squares method which is adapted to the identification of parameters in ecosystems with incomplete data; many models can be found in literature. The sentinel concept relies on the following three objects: some state equation (for instance (1)), some observation function (2), and some control function $u$ to be determined.

J.L.Lions calls a "sentinel", a functional $\mathcal{S}($.$) which is the scalar product of the measure y_{\text {obs }}$ and a function $u$. It is built to get some information on the pollution term.

\subsection{Presentation of the method}

Proposition 2.1. (definition, existence and uniqueness of the sentinel)

We now consider the sentinel method of Lions which is another attempt and brings better answer to question (q), as we will explain now

Let $h_{0}$ be a given function on $\left(L^{2}(\mathcal{O} \times] 0, T[)\right)^{n}$ such that

$$
h_{0} \geq 0, \int_{0}^{T} \int_{\mathcal{O}} h_{0} d x d t=1
$$

For any control function $u_{\epsilon} \in\left(L^{2}(\mathcal{U})\right)^{n}$, set

$$
\mathcal{S}(\lambda, \tau)=\int_{\mathcal{Q}}\left(h_{0}+u_{\epsilon}\right) \chi_{\mathcal{O}} y(x, t ; \lambda, \tau) d x d t
$$

The role of the function $u_{\epsilon}$ appears in the following definition. We shall say that $\mathcal{S}$ defines a weakly sentinel (for the system (1), (2) and definition of $\left.h_{0}\right)$ if there exists $u_{\epsilon}$ such that the functional $\mathcal{S}$ satisfies the following conditions for all $\epsilon>0$ there exists $u_{\epsilon} \in\left(L^{2}(\mathcal{U})\right)^{n}$ such as

$$
\begin{gathered}
u_{\epsilon} \in\left(L^{2}(\mathcal{U})\right)^{n}, \quad \text { of minimal norm } \\
\left|\frac{\partial}{\partial \tau} \mathcal{S}(0,0)\right| \leq \epsilon, \forall \widehat{y}_{0}
\end{gathered}
$$
$\left.u_{\epsilon}\right)$.

Then $\mathcal{S}(\lambda, \tau)$ defined by (3) (4) (5) exists and is unique (that means the existence and uniqueness of the function 
Proof. It will take two steps:

$1 /$ the conditions (4) (5) will be rewritten into a control problem,

$2 /$ an weakly controllability result will be proved,

\section{First step:}

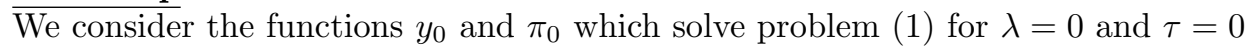

$$
\left\{\begin{array}{rllll}
\frac{\partial}{\partial t} y_{0}-\Delta y_{0}+y_{0} \nabla y_{0}+\nabla \pi_{0} & = & f & \text { in } & \mathcal{Q} \\
\operatorname{div} y_{0} & = & 0 & \text { in } & \mathcal{Q} \\
y_{0} & = & 0 & \text { on } & \Sigma \\
y_{0}(0) & = & y_{0} & \text { on } & \Omega
\end{array}\right.
$$

because of 3 we can write

$$
\mathcal{S}(0,0)=\int_{\mathcal{Q}}\left(h_{0}+u_{\epsilon}\right) \chi_{\mathcal{O}} y_{0}(x, t) d x d t
$$

is known. One carries out a development of Taylor of $\mathcal{S}$ in the vicinity of $(0,0)$

$$
\mathcal{S}(\lambda, \tau) \simeq \mathcal{S}(0,0)+\lambda \frac{\partial \mathcal{S}}{\partial \lambda}(0,0)+\tau \frac{\partial \mathcal{S}}{\partial \tau}(0,0), \quad \text { for } \quad \lambda, \tau \quad \text { small }
$$

and

and $y_{\lambda}(x, t)$ is the solution of

$$
\begin{aligned}
& \frac{\partial \mathcal{S}}{\partial \lambda}(0,0)=\int_{\mathcal{Q}}\left(h_{0}+u_{\epsilon}\right) \chi_{\mathcal{O}} y_{\lambda}(x, t) d x d t \\
& \frac{\partial \mathcal{S}}{\partial \tau}(0,0)=\int_{\mathcal{Q}}\left(h_{0}+u_{\epsilon}\right) \chi_{\mathcal{O}} y_{\tau}(x, t) d x d t
\end{aligned}
$$

$$
\left\{\begin{array}{rllll}
\frac{\partial}{\partial t} y_{\lambda}-\Delta y_{\lambda}+\nabla\left(y_{\lambda} \otimes y+y \otimes y_{\lambda}\right)+\nabla \pi_{\lambda} & = & \widehat{f} & \text { in } & \mathcal{Q} \\
\operatorname{div} y_{\lambda} & = & 0 & \text { on } & \mathcal{Q} \\
y_{\lambda} & = & 0 & \text { on } & \Sigma \\
y_{\lambda}(0) & = & 0 & \text { in } & \Omega
\end{array}\right.
$$

and $y_{\tau}(x, t)$ is the solution of

$$
\left\{\begin{array}{rllll}
\frac{\partial}{\partial t} y_{\tau}-\Delta y_{\tau}+\nabla\left(y_{\tau} \otimes y+y \otimes y_{\tau}\right)+\nabla \pi_{\tau} & = & 0 & \text { in } & \mathcal{Q} \\
\operatorname{div} y_{\tau} & = & 0 & \text { in } & \mathcal{Q} \\
y_{\tau} & = & 0 & \text { on } & \Sigma \\
y_{\tau}(0) & = & \widehat{y}_{0} & \text { on } & \Omega
\end{array}\right.
$$

To build the sentinel, one must determine $u_{\epsilon}$ which ensures the condition (4), (5) for a given positive $\epsilon$.

\subsubsection{Adjoint state}

Assume that $\frac{\partial y}{\partial \tau}$ can be defined for $\lambda=\tau=0$. Then, the $y_{\tau}$ solves the problem (8).

If $y_{\tau}$ and $y_{0}$ solve respectively (8) and (6), then the insensibility condition (5)

$$
\left|\frac{\partial \mathcal{S}}{\partial \tau}(0,0)\right|=\left|\int_{\mathcal{Q}}\left(h_{0}+u_{\epsilon}\right) \chi_{\mathcal{O}} y_{\tau}(x, t) d x d t\right| \leq \epsilon ; \forall \widehat{y}_{0},\left\|\widehat{y}_{0}\right\|_{L^{2}(\Omega)} \leq 1
$$

We set $\mathcal{D} q=\nabla q+\nabla q^{t}$ and introduce the adjoint state system associated to (8)

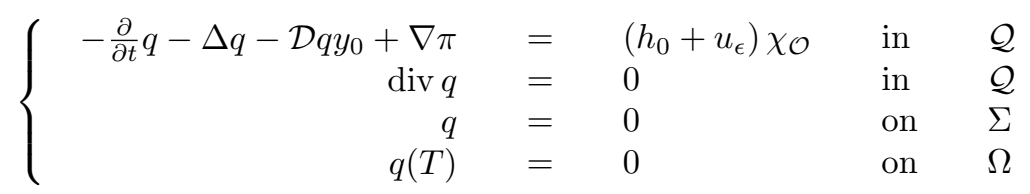

Therefore, let $\widehat{q}=q\left(u_{\epsilon}\right)$ be the unique solution, it is well known that $\widehat{q} \in L^{2}(0, T ; V) \cap C^{0}((0, T) ; H)$ depends on $u_{\epsilon}$ which is to be determined. 
Moreover, if we multiply the first equation in 10 by $y_{\tau}$, after integrating by parts over $\mathcal{Q}$, we obtain

$$
\left|\frac{\partial \mathcal{S}}{\partial \tau}(0,0)\right|=\left|\int_{\mathcal{Q}}\left(h_{0}+u_{\epsilon}\right) \chi_{\mathcal{O}} y_{\tau}(x, t) d x d t\right|=\left|\int_{\Omega} \widehat{y}_{0} \widehat{q}(0) d x\right|, \forall \widehat{y}_{0},\left\|\widehat{y}_{0}\right\|_{L^{2}(\Omega)} \leq 1
$$

This last equality combining with $(9)$ becomes

$$
\left|\int_{\Omega} \widehat{y}_{0} \widehat{q}(0) d x\right| \leq \epsilon, \forall \widehat{y}_{0},\left\|\widehat{y}_{0}\right\|_{L^{2}(\Omega)} \leq 1
$$

Consequently, insensibility condition (5) is valid if and only if

$$
\|\widehat{q}(0)\|_{L^{2}(\Omega)} \leq \epsilon
$$

The problem thus now to find $u_{\epsilon}$ in $\left(L^{2}(\mathcal{U})\right)^{n}$

Such that one has (11), and (5).

This is a controllability problem.

\subsubsection{Equivalent controllability problem}

For that one breaks up the system 10 into two systems

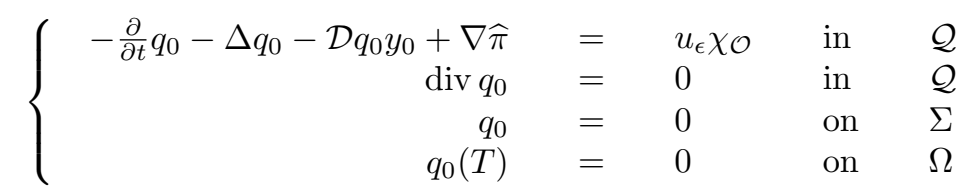

and

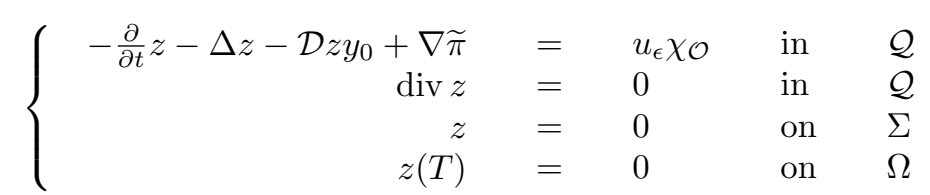

Thus $q=q_{0}+z$ such as $q_{0}$ is thus given. Then one seeks $u_{\epsilon}$ so that $z=z\left(u_{\epsilon}\right)$ who checks

$$
\left\|z\left(0 ; u_{\epsilon}\right)+q_{0}(0)\right\|_{L^{2}(\Omega)} \leq \epsilon
$$

If it is considered here that $u_{\epsilon}=$ function of control and $z=$ state of one (new) system.

That is to say $q_{0}(0)$ the desired state given by the resolution of the system 13 , the problem of regional controllability consists in finding, for all $\epsilon>0$ a control $u_{\epsilon}$ of the space of control $\left(L^{2}(\mathcal{U})\right)^{n}$ allowing to approach with $\epsilon$ meadows, in a time finished, the state $z(t)$ of the system 14 of an initial state $z(T)=0$, in a desired final state $q_{0}(0)$ on $\Omega$ (see: [11]).

\section{Second step:}

\subsubsection{Penalization and system of optimality}

For $\vartheta>0$, consider the function $J_{\vartheta}$ defined by

$$
J_{\vartheta}\left(u_{\epsilon}, z\right)=\frac{1}{2} \int_{0}^{T} \int_{\mathcal{O}} u_{\epsilon}^{2} d x d t+\frac{1}{2 \vartheta}\left\|-\frac{\partial}{\partial t} z-\Delta z-\mathcal{D} z y_{0}+\nabla \widetilde{\pi}-u_{\epsilon} \chi_{\mathcal{O}}\right\|_{L^{2}(\Omega \times] 0, T[)}^{2}
$$

Where one posed $z^{\prime}=\partial z / \partial t$

In 16 , one considers all $z$ such that

$$
\left\{\begin{array}{l}
-\frac{\partial}{\partial t} z-\Delta z-\mathcal{D} z y_{0}+\nabla \widetilde{\pi} \in L^{2}(\Omega \times(0, T)) \\
\operatorname{div} z=0 ; z(T)=0 ; z=0 \quad \text { on } \quad \Sigma \\
\left\|z\left(0 ; u_{\epsilon}\right)+q_{0}(0)\right\|_{L^{2}(\Omega)} \leq \epsilon
\end{array}\right.
$$


Let $u_{\epsilon}^{\vartheta}, z^{\vartheta}$ the solution of

$$
\inf J_{\vartheta}\left(u_{\epsilon}, z\right)
$$

One poses moreover

$$
\rho^{\vartheta}=\frac{1}{\vartheta}\left(-\frac{\partial}{\partial t} z^{\vartheta}-\Delta z^{\vartheta}-\mathcal{D} z^{\vartheta} y_{0}+\nabla \widetilde{\pi}-\chi_{\mathcal{O}} u_{\epsilon}^{\vartheta}\right)
$$

The couple $u_{\epsilon}^{\vartheta}, z^{\vartheta}$ is characterized by

$$
\iint_{\mathcal{O} \times(0, T)} u_{\epsilon}^{\vartheta} \cdot \widehat{u}_{\epsilon} d x d t+\iint_{\Omega \times(0, T)} \rho^{\vartheta}\left(-\frac{\partial}{\partial t} \widehat{z}-\Delta \widehat{z}-\mathcal{D} \widehat{z} y_{0}+\nabla \widetilde{\pi}-\chi_{\mathcal{O}} \widehat{u}_{\epsilon}\right) d x d t=0
$$

$\forall \widehat{u}_{\epsilon} \in\left(L^{2}(\mathcal{U})\right)^{n}$ and for all $\widehat{z}$ such that

$$
\left\{\begin{array}{l}
-\frac{\partial}{\partial t} \widehat{z}-\Delta \widehat{z}-\mathcal{D} \widehat{z} y_{0}+\nabla \widetilde{\pi}-\chi_{\mathcal{O}} \widehat{u}_{\epsilon} \in L^{2}(\Omega \times(0, T)) \\
\operatorname{div} \widehat{z}=0 \\
\widehat{z}(T)=0 ; \widehat{z}(0)=0, \widehat{z}=0 \quad \text { on } \quad \Sigma
\end{array}\right.
$$

One deduces from that

$$
\left\{\begin{aligned}
-\frac{\partial}{\partial t} \rho^{\vartheta}-\Delta \rho^{\vartheta}-\mathcal{D} \rho^{\vartheta} y_{0}+\nabla \sigma & =0 \\
\operatorname{div} \rho^{\vartheta} & =0
\end{aligned}\right.
$$

with

$$
\rho^{\vartheta}=0 \quad \text { on } \quad \Sigma
$$

without any information on $\rho^{\vartheta}$ with $t=0$ or with $T=0$, such that

$$
u_{\epsilon}^{\vartheta}=\chi_{\mathcal{O}} \rho^{\vartheta}
$$

\subsubsection{System of optimality}

Thus let us suppose, for the moment, that in a suitable topology, $\rho^{\vartheta} \longrightarrow \rho$, when $\vartheta \rightarrow 0$.

One has

$$
\left\{\begin{aligned}
-\frac{\partial}{\partial t} \rho-\Delta \rho-\mathcal{D} \rho y_{0}+\nabla \sigma & =0 \\
\rho & =0 \text { in } \Omega \times(0, T) \\
\rho(0) & =\rho^{0} \\
\operatorname{div} \rho=0 & =0
\end{aligned}\right.
$$

Where $\rho^{0}$ is not known for the moment.

One defines then $z$ by

$$
\left\{\begin{aligned}
-\frac{\partial}{\partial t} z-\Delta z-\mathcal{D} z y_{0}+\nabla \widetilde{\pi} & =\chi_{\mathcal{O}} \rho \\
\operatorname{div} z & =0 \\
z & =0 \\
z(T) & =0
\end{aligned} \text { on } \Sigma\right.
$$

Lastly, one must have 15

$$
\left\|z\left(0 ; u_{\epsilon}\right)+q_{0}(0)\right\|_{L^{2}(\Omega)} \leq \epsilon
$$

who is now an equation in $\rho^{0}$.

If, in a suitable space, $\rho^{0}$ exist and is single, then it $u_{\epsilon}$ sought is given by

$$
u_{\epsilon}=\chi_{\mathcal{O}} \rho
$$

The sentinel defined by $h_{0}$ is thus given by

$$
\int_{\mathcal{Q}}\left(h_{0}+\rho\right) \chi_{\mathcal{O}} y(x, t ; \lambda, \tau) d x d t
$$

Remain to solve (25), then to examine whether the sentinel is not identically null.

We now define a linear operator $\Lambda$ by

$$
\Lambda \rho^{0}=z(0)
$$


It remains to solve (25).

Multiplying (24) by $\rho$, we obtain after integrating by part

$$
\left\langle\Lambda \rho^{0}, \rho^{0}\right\rangle=\iint_{\mathcal{O} \times(0, T)} \rho^{2} d x d t
$$

What results in introducing

$$
\left\|\rho^{0}\right\|_{F}=\left(\iint_{\mathcal{O} \times(0, T)} \rho^{2} d x d t\right)^{\frac{1}{2}}
$$

One indicates by $F$ the space of Hilbert separate and supplemented regular functions $\rho^{0}$ for the norm (27).

$\Lambda \in \mathcal{L}\left(F, F^{\prime}\right)$ is an isomorphism of $F$ on $F^{\prime}$, and $\Lambda^{*}=\Lambda$;

$F^{\prime}$ being the dual space of $F$

The equation 25 is written $\Lambda \rho^{0}=-q_{0}(0)$

from where

$$
\rho^{0}=-\Lambda^{-1} q_{0}(0)
$$

subject checking that

$$
q_{0}(0) \in F^{\prime}
$$

But if one multiplies 13 by $\rho$, one sees that

$$
\left\langle q_{0}(0), \rho^{0}\right\rangle=\int_{\mathcal{Q}}\left(h_{0} \times \rho\right) \chi_{\mathcal{O}} d x d t
$$

Thus

$$
\left|\left\langle q_{0}(0), \rho^{0}\right\rangle\right| \leq\left\|h_{0}\right\|_{L^{2}(\mathcal{O} \times(0, T))}\left\|\rho^{0}\right\|_{F}
$$

from where 30 with $\left\|q_{0}(0)\right\|_{F^{\prime}} \leq\left\|h_{0}\right\|_{L^{2}(\mathcal{O} \times(0, T))}$

In what follows we apply the preceding result to estimate the term of pollution of the system (1).

\subsection{A use of the concept of sentinel: Detection of pollution}

Let us now, present a use of the concept of sentinel applied to some perturbed Navier-Stokes system.

Remark 2.2. If the semigroup $S^{*}(t)$ generated by the operator $A^{*}$ is compact in $L^{2}(\Omega)$, the system (14) is not exactly controllable (see [15]).

Remark 2.3. There are systems which are weakly controllable but they are not exactly controllable.

Example 2.4. $\Omega$ an open subset of $\mathbb{R}^{n}$ of smooth boundary $\partial \Omega$, we consider here the state equation

$$
\left\{\begin{array}{llll}
y^{\prime}-\Delta y & = & v & (x, t) \in \mathcal{Q} \\
y(x, 0) & = & 0 & \text { in } \Omega \\
y(x, t) & = & 0 & \text { on } \Sigma
\end{array}\right.
$$

The system above is a particular case of system (14); indeed, it is enough to take $A^{*}=\Delta$ when $y \in D(\Omega)=$ $H^{2}(\Omega) \cap H_{0}^{1}(\Omega), \mathcal{O}=\Omega, v=u_{\epsilon} \in L^{2}(\mathcal{U})$. This system cannot be exactly controllable in $L^{2}(\Omega)$ because the semigroup $S^{*}(t)$ generated by $A^{*}=\Delta$ is compact, but it is exactly controllable in $H_{0}^{1}(\Omega)$ (EL Jai, Pritchard [15]).

These two remarks led us to introduce the notion of the sentinel to estimate the term of pollution independently of the missing term. It is supposed that the system (14) is not exactly controllable thus the following theorem shows the interest of weakly controllability in the construction industry of the sentinels.

Theorem 2.5. If the system (14) is weakly controllable then for all $\epsilon$ positive it exists a function $u_{\epsilon} \in\left(L^{2}(\mathcal{U})\right)^{n}$ who checks the conditions (4), (5) of the "proposition 2.1."

Proof. its shows already. 
Theorem 2.6. Since the system (14) is weakly controllable on $\Omega$ then one has

$$
\int_{\mathcal{Q}} \widehat{q}\left(h_{0}\right)\{\lambda \widehat{f}\} d x d t \leq \int_{\mathcal{Q}}\left(h_{0}+u_{\epsilon}\right) \chi_{\mathcal{O}}\left|m_{0}-y_{0}\right| d x d t+\tau \epsilon
$$

where $y_{0}(x, t)$ is the solution of $(6)$ and $m_{0}$ is the state observed on $\mathcal{O}$ during the interval of time $(0, T)$.

Proof. that is to say $\mathcal{S}(\lambda, \tau)$ the sentinel defined by $h_{0}$ thus

$$
\lambda \frac{\partial \mathcal{S}}{\partial \lambda}(0,0)=\lambda \int_{\mathcal{Q}}\left(h_{0}+u_{\epsilon}\right) \chi_{\mathcal{O}} y_{\lambda}(x, t) d x d t=\mathcal{S}(\lambda, \tau)-\mathcal{S}(0,0)-\tau \frac{\partial \mathcal{S}}{\partial \tau}(0,0)
$$

And on the observatory $\mathcal{O}$ one poses $y=m_{0}$ then

$$
\lambda \frac{\partial \mathcal{S}}{\partial \lambda}(0,0)=\int_{\mathcal{Q}}\left(h_{0}+u_{\epsilon}\right) \chi_{\mathcal{O}}\left(m_{0}-y_{0}\right) d x d t-\tau \frac{\partial \mathcal{S}}{\partial \tau}(0,0)
$$

where $y_{\lambda}(x, t)$ is the solution of (7).

Now, we designate as $\widehat{q}\left(h_{0}\right)$ the unique solution of 10$)$ depending on $h_{0}$.

Multiplying (10) by $y_{\lambda}$, we obtain after integrating by part;

$$
\int_{\mathcal{Q}} \widehat{q}\left(h_{0}\right)\{\lambda \widehat{f}\} d x d t=\lambda \int_{\mathcal{Q}}\left(h_{0}+u_{\epsilon}\right) \chi_{\mathcal{O}} y_{\lambda}(x, t) d x d t
$$

and in addition one has

$$
\left|\frac{\partial \mathcal{S}}{\partial \tau}(0,0)\right|=\left|\int_{\mathcal{Q}}\left(h_{0}+u_{\epsilon}\right) \chi_{\mathcal{O}} y_{\tau}(x, t) d x d t\right| \leq \epsilon
$$

It results that the unknown pollution term $\lambda \widehat{f}$ can be defined as follows

$$
\begin{aligned}
\int_{\mathcal{Q}} \widehat{q}\left(h_{0}\right)\{\lambda \hat{f}\} d x d t & =\mathcal{S}(\lambda, \tau)-\mathcal{S}(0,0)-\tau \frac{\partial \mathcal{S}}{\partial \tau}(0,0) \\
& \leq \int_{\mathcal{Q}}\left(h_{0}+u_{\epsilon}\right) \chi_{\mathcal{O}}\left|m_{0}-y_{0}\right| d x d t+\tau \epsilon
\end{aligned}
$$

Thus, the proof of Theorem.

\section{3 Équivalence à un problème de contrôlabilité approchée à zéro}

La condition (5) sont équivalente à

$$
\left|\int_{\mathcal{Q}}\left(h_{0}+u_{\epsilon}\right) \chi_{\mathcal{O}} y_{\tau}(x, t) d x d t\right| \leq \epsilon
$$

En multipliant 10 par $y_{\tau}$ et intégrant par parties, on obtient

$$
\left|\frac{\partial \mathcal{S}}{\partial \tau}(0,0)\right|=\left|\int_{\mathcal{Q}}\left(h_{0}+u_{\epsilon}\right) \chi_{\mathcal{O}} y_{\tau}(x, t) d x d t\right|=\left|\int_{\Omega} \widehat{y}_{0} \widehat{q}(0) d x\right|
$$

Telle que $\widehat{q}=q\left(u_{\epsilon}\right) \in L^{2}(0, T ; V) \cap \mathcal{C}^{0}((0, T) ; H)$.

D'après $(11)-(5))$ on a

$$
\left|\int_{\Omega} \widehat{y}_{0} \widehat{q}(0) d x\right|=\left|\left(\widehat{q}(0), \widehat{y}_{0}\right)\right|
$$

Donc

$$
\|\widehat{q}(x, 0)\|_{L^{2}(\Omega)} \leq \epsilon
$$

Il s'agit là maintenant d'un problème du type "contrôlabilité approchée à zéro" 


\section{Existence d'une sentinelle approchée}

On rappelle que l'existence d'une sentinelle approchée se ramène à l'étude d'un problème d'optimisation sous contraintes

$$
(P):\left(\left\{\begin{array}{c}
\min \left\|u_{\epsilon}\right\|_{L^{2}} \\
\left(u_{\epsilon}, q\right) \in D
\end{array}\right)\right.
$$

Telle que

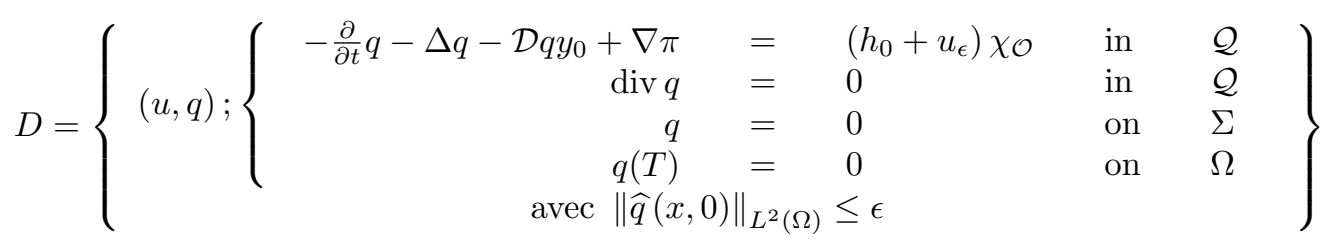

On utilise une autre approche différente basée sur un résultat important de continuation unique de MIZOHATA.

Theorem 4.1. Pour $\epsilon>0, h_{0} \in\left(L^{2}(\mathcal{O} \times(0 ; T))\right)^{n}$, il existe un contrôle $u_{\epsilon}$ et un état q tels que (10) et 112) sont vérifiées. En outre, il existe un unique couple $\left(\widehat{u}_{\epsilon}, \widehat{q}_{\epsilon}\right)$ avec $\widehat{u}_{\epsilon}$ de norme minimale dans $\left(L^{2}(\mathcal{O} \times(0 ; T))\right)^{n}, c$ 'est à dire tel que (10), (11) et (4) sont vérifiées.

Proof. (Théorème 4.1)

On introduit maintenant l'ensemble des états atteignables à l'instant $t=0$ défini par

$$
\mathcal{F}(0)=\left\{z\left(u_{\epsilon}, 0\right) \quad \text { telle que } \quad u_{\epsilon} \in\left(L^{2}(\mathcal{U})\right)^{n}\right\}
$$

$\mathcal{F}(0)$ est un espace vectoriel. Et pour démontrer la densité, il suffit d'utiliser le théorème de HAHN-BANACH et de remarquer que $\mathcal{F}^{\perp}$ est réduit à zéro.

Comme $\{0\} \subset \mathcal{F}^{\perp}(0)$, il reste à montrer que $\mathcal{F}^{\perp}(0) \subset\{0\}$. Soit $\rho^{0} \in \mathcal{F}^{\perp}(0)$, alors

$$
\left\langle\rho^{0}, z(0)\right\rangle_{L^{2}(\Omega)}=\int_{\Omega} \rho^{0} z(0) d x=0
$$

où $z$ est la solution de (14), il est donc naturel de définir l'adjoint $\rho$ de $z$, c'est la solution du problème (23).

Le système (18) est un problème classique de l'équation de la chaleur qui a une solution unique $\rho$.

Maintenant, on multiplie la première équation du système 23 par $z$. Après intégration par parties sur $\mathcal{Q}$, on obtient

$$
\iint_{\Omega \times(0, T)} \rho\left(L^{*} z+\nabla \widetilde{\pi}\right) d x d t-\int_{\Omega} \rho^{0} z(0) d x=0
$$

Avec $L^{*} z=-\frac{\partial}{\partial t} z-\Delta z-\mathcal{D} z y_{0}$

Ceci est équivalent à $\iint_{\Omega \times(0, T)} \rho u_{\epsilon} \chi_{\mathcal{O}} d x d t=0$. Parce que, $\rho^{0} \in \mathcal{F}^{\perp}(0)$ et $z(0) \in \mathcal{F}(0)$.

On désigne alors par $y_{0}$ une solution du système $(6), y_{0} \in L^{2}(0, T ; V) \cap L^{\infty}(0, T ; H)$.

On peut considérer ici $y \chi_{\mathcal{O}}=y_{\text {obs }}$ dans $L^{2}(\mathcal{O} \times(0, T))^{n} ; n \geq 2$.

- Pour $n=2$.

Dans le cas $y \chi_{\mathcal{O}}=\left\{y_{1} \chi_{\mathcal{O}}, y_{2} \chi_{\mathcal{O}}\right\}, H=L^{2}(\mathcal{O} \times(0, T))^{2}$; il s'agit d'une adaptation du théorème de MIZOHATA. Si $\rho_{1}=\rho_{2}=0 \operatorname{sur} \mathcal{O} \times(0, T)$ alors $\rho \equiv 0$.

$\mathrm{Au}$ moins si les coefficients $z_{0}$ sont assez réguliers (cf. J.C. SAUT et B. SCHEURER).

Dans le cas $y \chi_{\mathcal{O}}=y_{2} \chi_{\mathcal{O}}, H=L^{2}(\mathcal{O} \times(0, T))$, le problème correspondant semble ouvert. Considérons seulement le cas très particulier où $y_{0}=0$. Alors $\rho$ satisfait à l'équation de Stokes

$$
\rho^{\prime}-\mu \Delta \rho+\nabla \sigma=0 ; \operatorname{div} \rho=0 .
$$

Par analyticité, si $\rho_{2}=0$ sur $\mathcal{O} \times(0, T)$ il en résulte que $\rho_{2}=0$ dans $\Omega \times(0, T)$.

Mais alors $\operatorname{div} \rho=0$ implique $\frac{\partial \rho_{1}}{\partial x_{1}}=0$ dans $\Omega \times(0, T)$. Ce qui est impossible avec $\rho_{1}=0$ sur $\Gamma$ sauf si $\rho_{1}=0$ dans $\Omega \times(0, T)$ donc $\rho^{0}=0$.

- Pour $n=3$. 
Dans le cas $y_{0}=0$, pour la situation $y \chi_{\mathcal{O}}=\left\{y_{2} \chi_{\mathcal{O}}, y_{3} \chi_{\mathcal{O}}\right\}$ avec $H=L^{2}(\mathcal{O} \times(0, T))^{2}$. On a: $\rho_{2}=\rho_{3}=0$ sur $\mathcal{O} \times(0, T)$, alors, par analyticité, $\rho_{2}=\rho_{3}=0$ dans $\Omega \times(0, T)$. Alors comme div $\rho=0$, on a $\frac{\partial \rho_{1}}{\partial x_{1}}=0$ dans $\Omega \times(0, T)$, d'où $\rho_{1}=0$ (car $\rho_{1}=0$ au bord) et donc $\rho^{0}=0$.

La situation $y \chi_{\mathcal{O}}=y_{3} \chi_{\mathcal{O}}$ avec $H=L^{2}(\mathcal{O} \times(0, T))$, conduit à un problème intéressant. Soit donc $\rho$ vérifiant $\rho^{\prime}-\mu \Delta \rho+\nabla \sigma=0 ; \operatorname{div} \rho=0$ et $\rho_{3}=0$ sur $\mathcal{O} \times(0, T)$. Alors $\rho_{3}=0$ dans $\Omega \times(0, T)$. Cela implique-t-il que $\rho \equiv 0$. Alors on déduire, quel que soit $n$ si $\rho_{n}=0$ sur $\mathcal{O} \times(0, T)$, quand a-t-on $\rho \equiv 0$.

Enfin, nous avons $\rho \equiv 0$ et par conséquent $\rho^{0}=0$. Ce qui montre que $\mathcal{F}^{\perp}(0)=\{0\}$.

\section{Caractérisation du contrôle optimal}

On va caractériser le contrôle optimal en utilisant un résultat de dualité de FENCHEL-ROCKAFELLAR.

Le système d'optimalité satisfait par $\left(\widehat{u}_{\epsilon}, \widehat{q}_{\epsilon}\right)$ est établit comme suit.

Soit $\rho^{0} \in L^{2}(\Omega)$ et $\rho$ la solution associée de $(23)$.

On introduit maintenant la fonctionnelle $J_{\varepsilon}$ "coercive" définie par

$$
J_{\varepsilon}\left(\rho^{0}\right)=\frac{1}{2} \int_{0}^{T} \int_{\mathcal{O}}|\rho|^{2} d x d t+\epsilon\left\|\rho^{0}\right\|_{L^{2}(\Omega)}+\int_{0}^{T} \int_{\mathcal{O}} h_{0} \rho d x d t
$$

On considère le problème sans contrainte suivant:

$$
\left(P_{\varepsilon}\right): \mid \begin{gathered}
\min J_{\varepsilon}\left(\rho^{0}\right) \\
\rho^{0} \in L^{2}(\Omega)
\end{gathered}
$$

Alors, on a

Theorem 5.1. Le problème (32) a une solution unique $\widehat{\rho}^{0} \in L^{2}(\Omega)$. En outre, si $\widehat{\rho}$ est la solution de (23) associé $\grave{a} \widehat{\rho}^{0}$, alors $\left(\widehat{u}_{\epsilon}=\widehat{\rho}, \widehat{q}\right)$ est une solution telle que (10), (11) et (4) sont vérifiées.

Proof. Pour montrer que $J_{\varepsilon}$ est coercive. il suffit de montrer la relation suivante

$$
\lim _{\left\|\rho^{0}\right\|_{L^{2}(\Omega)} \rightarrow \infty} \frac{J_{\varepsilon}\left(\rho^{0}\right)}{\left\|\rho^{0}\right\|_{L^{2}(\Omega)}} \geq \epsilon .
$$

Comme $J_{\varepsilon}$ atteint sa valeur minimale à $\widehat{\rho}^{0} \in L^{2}(\Omega)$, alors, pour tout $\varphi^{0} \in L^{2}(\Omega)$ et tout $r \in \mathbb{R}$ on a

$$
J_{\varepsilon}\left(\widehat{\rho}^{0}\right) \leq J_{\varepsilon}\left(\hat{\rho}^{0}+r \varphi^{0}\right) \Longrightarrow J_{\varepsilon}\left(\hat{\rho}^{0}+r \varphi^{0}\right)-J_{\varepsilon}\left(\hat{\rho}^{0}\right) \geq 0
$$

D'autre part,

$$
\begin{aligned}
J_{\varepsilon}\left(\widehat{\rho}^{0}\right)=\frac{1}{2} \int_{0}^{T} & \int_{\mathcal{O}}|\widehat{\rho}|^{2} d x d t+\epsilon\left\|\widehat{\rho}^{0}\right\|_{L^{2}(\Omega)}+\int_{0}^{T} \int_{\mathcal{O}} h_{0} \widehat{\rho} d x d t \\
J_{\varepsilon}\left(\widehat{\rho}^{0}+r \varphi^{0}\right) & =\left.\frac{1}{2} \int_{0}^{T} \int_{\mathcal{O}}\left|\widehat{\rho}^{2} d x d t+\frac{r^{2}}{2} \int_{0}^{T} \int_{\mathcal{O}}\right| \varphi\right|^{2} d x d t \\
& +r \int_{0}^{T} \int_{\mathcal{O}} \widehat{\rho} \varphi d x d t+\sqrt{\varepsilon}\left\|\widehat{\rho}^{0}+r \varphi^{0}\right\|_{L^{2}(\Omega)} \\
& +\int_{0}^{T} \int_{\mathcal{O}} h_{0}(\widehat{\rho}+r \varphi) d x d t
\end{aligned}
$$

En substituant (34) dans (33) et après les simplifications, on trouve

$$
\begin{aligned}
0 & \leq J_{\varepsilon}\left(\hat{\rho}^{0}+r \varphi^{0}\right)-J_{\varepsilon}\left(\widehat{\rho}^{0}\right) \\
0 & \leq \frac{r^{2}}{2} \int_{0}^{T} \int_{\mathcal{O}}|\varphi|^{2} d x d t+\epsilon\left[\left\|\hat{\rho}^{0}+r \varphi^{0}\right\|_{L^{2}(\Omega)}-\left\|\hat{\rho}^{0}\right\|_{L^{2}(\Omega)}\right] \\
& +r\left[\int_{0}^{T} \int_{\mathcal{O}} \hat{\rho} \varphi d x d t+\int_{0}^{T} \int_{\mathcal{O}} h_{0} \varphi d x d t\right]
\end{aligned}
$$


D'autre part,

$$
\left\|\hat{\rho}^{0}+r \varphi^{0}\right\|_{L^{2}(\Omega)}-\left\|\hat{\rho}^{0}\right\|_{L^{2}(\Omega)} \leq|r| \cdot\left\|\varphi^{0}\right\|_{L^{2}(\Omega)}
$$

De 36 et 35 , on obtient pour tous $\varphi^{0} \in L^{2}(\Omega)$ et $r \in \mathbb{R}$,

$$
\begin{aligned}
0 & \leq \frac{r^{2}}{2} \int_{0}^{T} \int_{\mathcal{O}}|\varphi|^{2} d x d t+\epsilon|r| \cdot\left\|\varphi^{0}\right\|_{L^{2}(\Omega)} \\
& +r\left[\int_{0}^{T} \int_{\mathcal{O}} \widehat{\rho} \varphi d x d t+\int_{0}^{T} \int_{\mathcal{O}} h_{0} \varphi d x d t\right]
\end{aligned}
$$

En divisant par $r>0$ et en passant à la limite $r \rightarrow 0$, on obtient

$$
\epsilon \cdot\left\|\varphi^{0}\right\|_{L^{2}(\Omega)}+\int_{0}^{T} \int_{\mathcal{O}} \widehat{\rho} \varphi d x d t+\int_{0}^{T} \int_{\mathcal{O}} h_{0} \varphi d x d t \geq 0
$$

Les mêmes calculs avec $r<0$ donnent

$$
\left|\int_{0}^{T} \int_{\mathcal{O}} \widehat{\rho} \varphi d x d t+\int_{0}^{T} \int_{\mathcal{O}} h_{0} \varphi d x d t\right| \leq \epsilon\left\|\varphi^{0}\right\|_{L^{2}(\Omega)} ; \forall \varphi^{0} \in L^{2}(\Omega) .
$$

Donc,

$$
\left|\int_{0}^{T} \int_{\mathcal{O}} h_{0} \varphi d x d t+\int_{0}^{T} \int_{\mathcal{O}} \widehat{\rho} \varphi d x d t\right| \leq \epsilon\left\|\varphi^{0}\right\|_{L^{2}(\Omega)} ; \forall \varphi^{0} \in L^{2}(\Omega) .
$$

Donc,

$$
\left|\int_{0}^{T} \int_{\mathcal{O}}\left(h_{0}+\widehat{\rho}\right) \varphi d x d t\right| \leq \epsilon\left\|\varphi^{0}\right\|_{L^{2}(\Omega)} ; \forall \varphi^{0} \in L^{2}(\Omega) .
$$

Aussi, si on prend $\widehat{u}_{\epsilon}=\widehat{\rho}$ dans 10 et on multiplie la première équation du système (10) par $\varphi$ solution de (23), on obtient après intègration par parties $\operatorname{sur} \mathcal{Q}$,

$$
\int_{\Omega} q(0) \varphi^{0} d x=\int_{0}^{T} \int_{\mathcal{O}}\left(h_{0}+\widehat{u}_{\epsilon}\right) \varphi d x d t
$$

On obtient des deux dernières relations

$$
\left|\int_{\Omega} q(0) \varphi^{0} d x\right| \leq \epsilon\left\|\varphi^{0}\right\|_{L^{2}(\Omega)} ; \forall \varphi^{0} \in L^{2}(\Omega) .
$$

Par conséquent,

$$
\|q(x, 0)\|_{L^{2}(\Omega)} \leq \epsilon .
$$

On a donc démontré le théorème suivant

Theorem 5.2. On suppose que les données manquantes sont dans les conditions initiales, et que la pollution apparaît au source.

On suppose que l'observatoire $\mathcal{O} \subset \Omega$. pour $h_{0}$ donné dans $L^{2}(\mathcal{O} \times(0, T))$ il existe une sentinelle, et une seule, portée par $\mathcal{O} \times(0, T)$. Elle est construite comme suit.

Pour $\rho^{0}$ donné, on définit $\rho$ par (23). Puis on définit z par (14). On définit alors $\rho^{0}$ par (23), où $q_{0}$ est définie par (13). Cela définit $\rho$ et donne la sentinelle

$$
\int_{0}^{T} \int_{\mathcal{O}}\left(h_{0}+\rho\right) y(\lambda, \tau) d x d t
$$




\section{Concluding remarks}

In this paper we have presented an efficient method to estimate the pollution terms in the Navier-Stokes system with missing initial data condition and perturbed term or pollution term. The theory used for the identification needs the sentinels method by Lions [12. And finally, we give the characterization of the weakly sentinel, which permits to identify the pollution parameters.

ACKNOWLEDGEMENTS. The authors thank the referees for their careful reading and their precious comments. Their help is much appreciated.

\section{References}

[1] I.Rezzoug, A.Ayadi, Controllability for the parabolic equations. PJM, Vol. 6(2), 638-648. (2017).

[2] I.Rezzoug, Étude théorique et numérique des problèmes d'identification des systèmes gouvernés par des équations aux dérivées partielles, Thèse de doctorat, Université de Oum El Bouaghi, Algérie. (2014).

[3] I. I.Rezzoug, A.Ayadi, Identification of an unknown part of the boundary of an Navier-Stokes system by punctual sentinel. Sciences $\&$ Technologies A - No 35, Juin, pp. 31-35. (2012).

[4] Y.Miloudi, O.Nakoulima, A.Omrane, On the instantaneous sentinels in pollution problems of incomplete data, Inverse Problems in Science and Engineering, 17(4), (2009), $451-459$. https://doi.org/10.1080/17415970802015948 https://doi.org/10.1080/17415970802015948

[5] I.Rezzoug, Identification d'une partie de la frontière inconnue d'une membrane, Thèse de magister, Université de Oum El Bouaghi, Algérie. (2009).

[6] E.H.Zerrik, A.Afifi, A.Eijai, Systèmes dynamiques, Analyse régionale des systèmes linéaires distribués. Tome 2, Presses Universitaires de Perpignan. (2008).

[7] O.Nakoulima, A revision of J.L.Lions notion of sentinels, Portugal. Math. (N.S). Vol. 65, Fasc. 1, pp 1-22. (2008). https://doi.org/10.4171/pm/1795

[8] J.Velin, Discriminating distributed sentinel involving a navier stokes problem and parameter identi-cation, Esaim, Proseedings, 143-166. (2007). https://doi.org/10.1051/proc:071709

[9] Y.Miloudi, O.Nakoulima, A.Omrane, A Method for Detecting Pollution in Dissipative Systems with Incomplete Data, ESAIM: Proceedings, 17(2007) 67-79. https://doi.org/10.1051/proc:071706 https://doi.org/10.1051/proc:071706

[10] M.Clerc., P.L.Tallec, M.Mallet, Contrôle optimale de Navier-Stokes Parabolisé, Rapport de recherche, N 2653. (1995).

[11] E.H.Zerrik, Analyse régionale des systèmes distribués. Thèse. Univ. Mohammed V. Maroc. (1993).

[12] J.L.Lions, Sentinelles pour les systèmes distribués à données incomplètes. Masson, Paris. (1992).

[13] J.L.Lions, Contrôlabilité exacte, stabilisation et perturbations des systèmes distribués, Vol. 1. Contrôlabilité Exacte. Masson, Paris (1988). https://doi.org/10.1090/s0273-0979-1990-15909-9

[14] E.Zuazua, Contrôlabilité exacte de systèmes d'évolution non linéaires. C.R. Acad. Sci. Paris, 306, p. 129-132. (1988).

[15] A.EL Jai, A.J.Pritchard,Capteurs et actionneurs dans l'analyse des systèmes distribués. Masson. RMA 3. Paris. (1986). 
[16] R.Ttemam, Navier-stokes Equations. Theory and numerical analysis, Stud. Math. Appl., North-Holland, Amsterdam-New York-Oxford. (1984).

[17] R.Ttemam, Navier-stokes Equations. Theory and numerical analysis, Stud. Math. Appl., North-Holland, Amsterdam-New York-Oxford. (1984).

[18] A.V.Fursikov, Problèmes de contrôle et résultats sur la résolution unique de problèmes mixtes pour les équations de Navier-Stokes et Euler tridimensionnelles. Mat. Sbornik, 115 (157), p. 281-306. (1981).

[19] J.L.Lions, G.Prodi, Un théorème d'existence et unicité dans les équations de Navier-Stokes en dimension 2. C.R. Acad. Sci. Paris, 248, p. 3519-3521. (1959).

[20] O.A.Ladyzenskaya, Global solution of the boundary value problem for Navier-Stokes equations in 2 space dimensions. Doklady Akad. Nauk. SSSR, 123, p. 427-429. (1958).

[21] Z.Mizohata, Unicité du prolongement des solutions pour quelques opérateurs différentiels paraboliques, Mem. Coll. Sc. Univ. Kyoto, Série A, Vol 31, 219-239. (1958). 